\title{
Therapeutics
}

\section{Review: atypical antipsychotics and psychosocial interventions, alone or in combination, may reduce youth aggression}

Schur SB, Sikich L, Findling RL et al. Treatment recommendations for the use of antipsychotics for aggressive youth (TRAAY). Part 1: a review. J Am Acad Child Adolec Psychiatry, 2003 Feb;42:132-44.

QUESTION: How safe and effective are pharmacological and non-pharmacological treatments for aggression in children and adolescents?

\section{Design}

Systematic review with narrative synthesis.

\section{Data sources}

The reviewers searched Medline and PsycInfo from 1984 for data on atypical antipsychotics and from 1990 for other interventions. Information from presentations at scientific meetings was also included.

\section{Study selection}

Double blind, placebo controlled studies of atypical antipsychotics for aggression were eligible, as were studies of any design on other pharmacological agents and psychosocial interventions for aggression. Case reports and adult literature on the safety of atypical antipsychotics were used where controlled data for youth were lacking.

\section{Data extraction}

The authors do not provide details of how data were extracted for the review.

\section{Main results}

There are few data from controlled studies on treating aggression in children and adolescents. There is some evidence that psychosocial interventions may be effective alone or combined with pharmacological treatments. Psychotropic agents such as stimulants, mood stabilisers, and beta-blockers may also have some efficacy in reducing aggression. Antipsychotics, particularly atypical antipsychotics, are effective for reducing aggression in selected paediatric populations. Atypical antipsychotics are associated with fewer extrapyramidal symptoms compared with typical antipsychotics.

\section{Conclusions}

Psychosocial interventions and atypical antipsychotics may help reduce aggression in children and adolescents. Double blind studies are needed to assess the safety and efficacy of different atypical antipsychotics compared with each other and alternative medications.

\section{COMMENTARY}

Aggression in childhood and adolescence is a significant predictor of psychiatric consultation, referral to mental health services, prescription of psychotropics and hospitalisation. Yet aggression is a greatly under-researched area of childhood psychopathology. Although there are limited data on the efficacy of psychosocial and pharmacological interventions for aggression, Schur et al highlight two points. First, some psychosocial interventions seem to be more promising than others. The central component of promising interventions appears to be that they are well structured and based on the principles of learning theory.

Second, atypical antipsychotics have been assessed in a number of well designed placebo controlled trials in children with disruptive behaviour disorders. Atypical antipsychotics, especially risperidone, have been found to be effective and safe when prescribed at relatively low dosages of $0.5-2.0 \mathrm{mg}$ per day. Atypical antipsychotics seem to offer clear advantages above conventional antipsychotics, especially regarding extrapyramidal adverse effects.

A number of unanswered questions remain. First, it is important to extend the knowledge base with more methodologically sound studies, including head to head comparisons of different atypical antipsychotics and of atypical antipsychotics and mood stabilisers. In this review and the accompanying guidelines, ${ }^{1}$ the authors focus particularly on impulsive or affective types of aggression, but few data exist on the reliability and validity of distinctions between impulsive and predatory aggression. There are no accepted instruments to allow clinicians to make the distinction. Further, there is a lack of data about the relative efficacy of psychosocial and pharmacological interventions, and about the added value of combined treatment approaches, although in usual care most clinicians would tend to offer a menu of psychosocial and medical interventions when managing severe aggression in youth. Finally, we must appreciate that aggression is a symptom that may be observed in the context of various categorical diagnoses ranging from oppositional defiant disorder and conduct disorder through ADHD to posttraumatic stress disorder, mood disorders, bipolar disorder and psychosis. It is therefore of great importance that we improve our insight into the modifying and moderating role of categorical diagnoses in the efficacy and safety of treatments for aggression. Jan K Buitelaar, MD PhD Department of Psychiatry University Medical Center, Nijmegen The Netherlands

1 Pappadopulos E, MacIntyre JC, Crismon ML et al. Treatment recommendation for the use of antipsychotics for aggressive youth (TRAAY). Part II. J Am Acad Child Adolesc Psychiatry 2003, 42: 145-61.
Carmel Hill Fund:

New York State Office of Mental Health: Center for the Advancement of Children's Mental Health.

For correspondence: PS Jensen, Center for the Advancements of Children's Mental Health, Columbia University, New York, 